\title{
Fortalecimiento de las competencias matemáticas a partir de la resolución de problemas en operaciones básicas de números naturales, para estudiantes del grado sexto en Colombia
}

\author{
Strengthening of mathematical competences from the resolution of problems in basic operations of \\ natural numbers, for sixth grade students in Colombia
}

\section{Fortalecimento de competências matemáticas a partir da resolução de problemas em operações básicas de números naturais, para estudantes do sexto ano na Colômbia}

\author{
María Alejandra Sánchez-Díaz ${ }^{a^{*}}$ \\ Licenciatura en matemáticas, Institución Educativa Colegio San José. Cúcuta, Colombia. \\ Orcid: 0000-0001-9278-9472
}

Forma de citar: Sánchez, M. (2017). Fortalecimiento de las competencias matemáticas a partir de la resolución de problemas en operaciones básicas de números naturales, para estudiantes del grado sexto en Colombia. Eco matemático 8(1). 62-70

Recibido: septiembre 3 de 2016

Aceptado: noviembre 28 de 2016

\section{Palabras clave}

Competencias matemáticas, Resolución de problemas, Operaciones básicas de Números Naturales.

\section{Keywords}

Mathematical competences, Problem solving, Basic Operations of Natural Numbers.

\begin{abstract}
Resumen: A continuación, se describe en el siguiente artículo, referente a una investigación realizada cuyo objetivo es implementar talleres guías para el fortalecimiento de la competencia matemática de resolución en los sistemas numéricos a través del Método Polya, para los estudiantes de sexto grado de la Institución Educativa Colegio San José "El Trigal” de Cúcuta. La metodología empleada fue de investigación acción, donde se utilizaron como instrumentos para recabar la información: la observación directa, diarios de campo, prueba diagnóstica y talleres guía. Con respecto a la propuesta, su autora presenta diversas estrategias de aplicación de talleres guía, con el fin de atender la dificultad que tienen los estudiantes del grado sexto para la resolución de problemas: que consiste en leer, analizar y solucionar situaciones problemáticas en los distintos conjuntos de numeración, reflejadas en los hallazgos obtenidos en las pruebas institucionales y en las llamadas "PRUEBAS SABER". Los resultados de la investigación determinaron que los participantes logran solucionar situaciones problemas, desde la Comprensión del enunciado del problema, Aclaración de dudas, Identificación de todos los datos y de lo que quería encontrar, Formulación de un plan para encontrar una solución, Realización de las operaciones correctamente y Verificación del resultado. Finalmente se propone a la Institución asignar una hora de Formulación y Solución de Problemas matemáticos, además la planeación de clases a partir de Talleres Guías, ya que facilitan la construcción de conocimientos a partir de aprendizajes significativos y la interacción con el otro.
\end{abstract}

Abstract: Next, it is described in the following article, referring to a research carried out whose objective is to implement guides workshops for the strengthening of the mathematical competence of resolution in the numerical systems through the Polya Method, for the sixth grade students of the Educational Institution Colegio San José "El Trigal" of Cúcuta. The methodology used was action research, where they were used as instruments to collect the information: direct observation, field diaries, diagnostic test and guide workshops. With respect to the proposal, the author presents

\footnotetext{
* Autor para correspondencia msanchez89@unab.edu.co
} 


\section{Palavras-chave}

Competencias Científicas, Física, Estudiantes. several strategies for the application of guiding workshops, in order to address the difficulty of sixth grade students to solve problems: reading, analyzing and solving problematic situations in different sets of numbers, reflected in the findings obtained in the institutional tests and in the so-called "TESTS KNOW". The results of the research determined that the participants managed to solve problem situations, from the understanding of the problem statement, clarification of doubts, identification of all the data and what he wanted to find, formulation of a plan to find a solution, operations correctly and Verification of the result. Finally it is proposed to the Institution to assign an hour of Formulation and Solution of Mathematical Problems, besides the planning of classes from Workshops Guides, since they facilitate the construction of knowledge from significant learning and interaction with the other.

Resumo: A continuação descreve-se sobre o assunto, com base em uma investigação realizada com o objetivo de implementar altos níveis para o fortalecimento da competência matemática de resolução nos sistemas numéricos e transversais de Polia, para os estudantes de sexto grau da Instituição Educativa de Colégio San José "El Trigal" de Cúcuta. A metodologia utilizada para investigar a situação, utiliza se como instrumental para reciclar a informação: a observação directa, diarios de campo, diagnósticos prueba y talleres guía. Con respeito a la propuesta, sua autora presenta várias estrategias de aplicação de talleres guía, con fin to de la dificultad que tienen os estudiantes del grado sexto para la resolución de problemas: que consiste em analisar, analisar e resolver situaciones problemáticas en los distintos Conjuntos de Numeração, Reflejadas e Ensaios Funcionais nas Instituições Institucionais e nas Llamadas "PRUEBAS SABRE". Los resultados da investigação sobre a situação dos problemas em situações difíceis, desde a Compromisso do enunciado do problema, Definição de dudas, Identidade de todos os dados e resultados da consulta, Formulário de plano para encontrar uma solução, Realização das operaciones correctamente y Verificación del resultado. Finalmente, propondo-se à criação de uma hora de formação e solução de recursos matemáticos, além da planificação de tarefas a partir de Talleres Guías, que facilitam a construção de centros a partir de aprendizados e da interacção com o otro.

\section{Introducción}

Las matemáticas comprenden un área del saber elemental para el proceso de formación estudiantil y su desarrollo lógico aritmético, es por ello que en muchos años se han realizado diversos estudios, a fin de mejorar los procesos de enseñanza para fortalecer diversas competencias matemáticas.

En la constante práctica del quehacer pedagógico se han podido definir ciertas dificultades en el proceso de enseñanza aprendizaje en la asignatura de matemáticas sobre todo en la resolución de problemas en los diferentes conjuntos numéricos, lo que conlleva a la innovación, actualización e inclusión de la lúdica y la tecnología en sus prácticas pedagógicas.

La mayor falencia presente en los estudiantes del grado sexto del Colegio San José "El Trigal" es la dificultad que tienen para la resolución de problemas: leer, analizar y solucionar situaciones problemáticas en los distintos conjuntos de numeración, la cual se ve reflejada en los resultados obtenidos en las pruebas institucionales y en las llamadas "PRUEBAS SABER".

Estas PRUEBAS SABER tienen como objetivo "visualizar el estado de las competencias y aprendizajes en matemáticas y lenguaje en los establecimientos educativos de acuerdo con los resultados en las PRUEBAS SABER $3^{\circ}, 5^{\circ} \mathrm{y}$ $9^{\circ}$, haciendo énfasis en aquellos aprendizajes en los que se deben realizar acciones pedagógicas para el mejoramiento". Presentan tres reportes, el primero explica el comportamiento general de la competencia asociada a cada prueba, comparando el 
resultado de la institución educativa con la Entidad Territorial Certificada y Colombia; la segunda describe el estado general de los aprendizajes de la competencia asociada, inclinando el número de aprendizajes que se encuentran en rojo, naranja, amarillo y verde. Los colores del semáforo están asociados al porcentaje de estudiantes que NO respondió correctamente los ítems de cada aprendizaje; y el tercero enumera los aprendizajes en los que es necesario implementar acciones de mejora con especial prioridad.

De acuerdo a esta breve explicación e inclinándose en la competencia: Resolución, referente al problema descrito, se tiene que en el INFORME POR COLEGIO 2017, PRUEBAS SABER $3^{\circ}$, $5^{\circ} \mathrm{Y} 9^{\circ} \mathrm{DE} 2016$ el $36 \%$ de los estudiantes no contestaron correctamente las preguntas

\section{Descripción general de la competencia.}

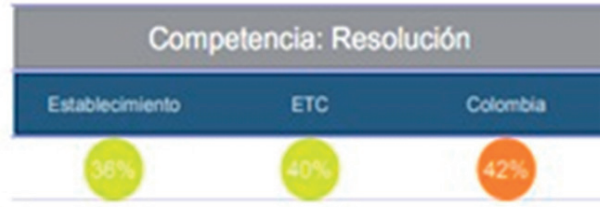

\section{Interpretación}

El 36\% do los estudiontes NO contestb correctomente las proguntas do esta compotencia.

Gráfica 1. Descripción general de la competencia de Resolución.

Fuente: MEN. Informe por Colegio 2017. Colombia Aprende.

Y de acuerdo a los aprendizajes evaluados en esta competencia la institución tiene un $0 \%$ de aprendizajes en rojo, el $33 \%$ en naranja, el 50\% en amarillo y $17 \%$ en verde. (Rojo: el $70 \%$ de los estudiantes de la institución no contestaron correctamente las preguntas relacionadas con el aprendizaje; Naranja: entre el $40 \%$ y el $69 \%$ de los estudiantes del establecimiento No contestaron las preguntas relacionadas con el aprendizaje; Amarillo: entre el 20\% y el 39\% de los estudiantes del establecimiento No contestaron las preguntas relacionadas con el aprendizaje y Verde: el 19\% o menos de los estudiantes del establecimiento No contestaron las preguntas relacionadas con el aprendizaje), lo que quiere decir que en su mayoría hay dificultad en esta competencia.

\section{Descripción general de los aprendizajes.}
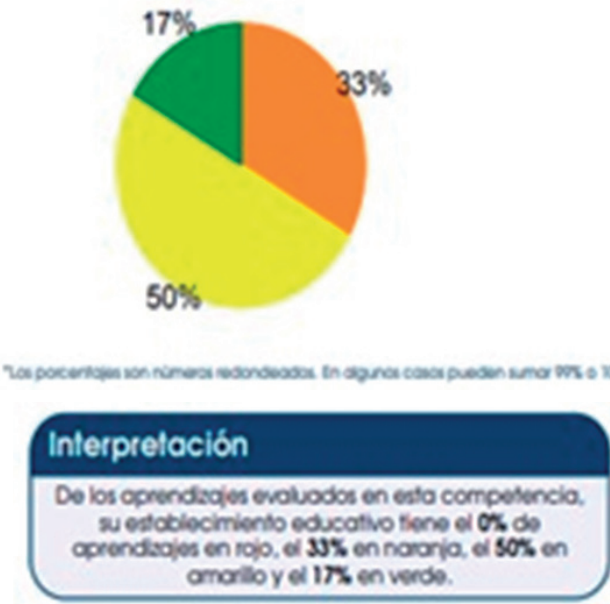

Gráfica 2. Descripción general de los aprendizajes en la competencia de Resolución.

Fuente: MEN. Informe por Colegio 2017. Colombia Aprende.

Por lo tanto, se debe implementar acciones pedagógicas de mejoramiento para los que están en el color rojo y naranja. Aquellos aprendizajes a mejorar según el INFORME DEL COLEGIO 2017 son:

E1 56\% de los estudiantes no resuelve ni formula problemas que requieren de la fracción como parte de un todo, como cociente y razón. El 47\% de los estudiantes no resuelve problemas que requieren representar datos relativos al entorno usando una o diferentes representaciones. El 39\% de los estudiantes no usa representaciones geométricas ni establece relaciones entre ellas para solucionar problemas.

El 34\% de los estudiantes no resuelve ni formula problemas multiplicativos rutinarios y no rutinarios de adición repetida, factor multiplicante, razón y producto cartesiano. E1 33\% de los estudiantes no resuelve ni formula problemas sencillos de proporcionalidad directa e inversa. El 14\% de los estudiantes no resuelve que requieren encontrar y/o dar significado a la medida de tendencia central de un conjunto de datos. 


\section{Aprendizajes.}

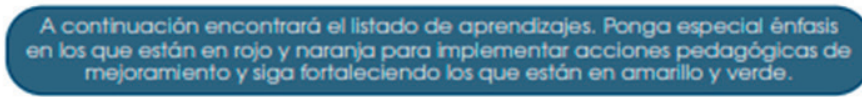

\section{Interpretación}

El $56 \%$ de los estudiantes NO contestó correctamente las preguntas correspondientes al primer aprendizaje.
Esta interpretación aplica de igual manera para los demás aprendizajes.

no resuelve ni formula problemas que requieren el uso de la fracción como parte de un todo, como cociente y como razón.

no resuelve problemas que requieren representar datos relativos al entorno usando una o diferentes representaciones.

no usa representaciones geométricas ni establece relaciones entre ellas para solucionar problemas.

no resuelve ni formula problemas multiplicativos rutinarios y no rutinarios de adición repetida, factor multiplicante, razón y producto cartesiano.

no resuelve ni formula problemas sencillos de proporcionalidad directa e inversa.

Gráfica 3: Aprendizajes en la competencia de Resolución.

Fuente: MEN. Informe por Colegio 2017. Colombia Aprende

\section{Materiales y método}

La investigación como tal hace que el docente en su quehacer pedagógico sea un investigador constante, un indagador de las posibles soluciones a las problemáticas que se presentan en el proceso de enseñanza aprendizaje, que su práctica pedagógica integrada con la reflexión y el análisis de las diferentes experiencias pedagógicas conlleven a generar propuestas de mejoramiento y transformación en su labor docente. Es decir, como dice Tamayo (1994), "El punto de partida del científico es la realidad, que mediante la investigación le permite llegar a la ciencia. El científico observa, descubre, explica y predice aquello que lo lleva a un conocimiento de la realidad". Lo cual permite indicar que este proyecto está orientado hacia una investigación de enfoque cualitativo. Según Tylor y Bogdan (1986), la investigación cualitativa es "aquella que produce datos descriptivos, las propias palabras de las personas, habladas o escritas, y la conducta observable".

El presente proyecto es de tipo investigación acción, que supone la enseñanza como un proceso de investigación hacia la constante búsqueda, la cual se ajusta mejor al perfil del docente como investigador, integrando la reflexión y el trabajo intelectual en el análisis de las experiencias educativas mejorando, innovando, comprendiendo los contextos educativos como meta de la calidad educativa y entendida como el proceso para estudiar, comprender y transformar el quehacer pedagógico a través de una formación adquirida durante la investigación: epistemológica, teórica, metodológica y estratégica.

Según Elliott (1993) define la investigación acción como "un estudio de una situación social con el 
fin de mejorar la calidad de la acción dentro de la misma", entendida como la reflexión de las prácticas pedagógicas en la que el docente y estudiante son participes y conlleve esta al mejoramiento y transformación de aquellas situaciones problemas que se presentan partiendo de la comprensión de un diagnóstico. Es decir, Elliot contempla a la investigación como una reflexión diagnóstica de la práctica.

Kemmis (1984) la investigación acción como ciencia práctica, moral y ciencia critica, la define: “... una forma de indagación autorreflexión realizada por quienes participan (profesorado, alumnado, o dirección por ejemplo) en las situaciones sociales (incluyendo las educativas) para mejorar la racionalidad y la justicia de: a. sus propias prácticas sociales o educativas; b. su comprensión sobre las mismas; y c. las situaciones e instituciones en que estas prácticas se realizan (aulas o escuelas, por ejemplo)", pensada como un proceso de reflexión en el ámbito educativo, llevando a cabo tres momentos, en primer lugar se define con certeza el problema, en segundo lugar se especifica un plan de acción y en tercer lugar se evalúa para comprobar y establecer la efectividad de la acción tomada, permitiendo el mejoramiento y transformación de la práctica pedagógica.

La investigación acción no solo es un proceso que permite transformar las prácticas del docente, sino también es un proceso que plantea un cambio social que colectivamente y a través de una metodología oriente hacia el cambio educativo. El modelo del proceso de la investigación acción inicia con la Identificación del problema; Construcción de un plan estratégico; ejecución del plan; evaluar el plan y verificar si permitió la solución del problema planteado, en caso contrario se debe volver a la construcción del plan estratégico hasta encontrar la solución al problema.

En el presente estudio se parte del análisis de los resultados en las PRUEBAS SABER $3^{\circ}, 5^{\circ}$ y $9^{\circ}$, especialmente al grado quinto, ya que este proyecto está dirigido a estudiantes de grado sexto de la institución, además un diagnóstico en donde se especifica el nivel en que se encuentran los estudiantes en la resolución de problemas. Se diseña y ejecuta una propuesta pedagógica que permita solventar las dificultades que presentan los estudiantes en la resolución de problemas matemáticos y finalmente se evaluará con el fin de establecer si se fortaleció la competencia matemática de resolución en los sistemas numéricos, a través del método Polya.

\section{El Método de Cuatro Pasos de Polya}

Este método está enfocado a la solución de problemas matemáticos, por ello parece importante señalar alguna distinción entre "ejercicio" y "problema". Para resolver un ejercicio, el estudiante aplica un procedimiento rutinario que lo lleva a la respuesta. Para resolver un problema, hace una pausa, reflexiona y hasta puede ser que ejecute pasos originales que no había ensayado antes para dar la respuesta. Esta característica de dar una especie de paso creativo en la solución no importa que tan pequeño sea, es lo que distingue un problema de un ejercicio.

Sin embargo, es prudente aclarar que esta distinción no es absoluta; depende en gran medida del estadio mental del estudiante que se enfrenta a ofrecer una solución: Para un niño pequeño puede ser un problema encontrar cuánto es el resultado de una suma simple. O bien, para niños de los primeros grados de primaria responder a la pregunta ¿Cómo repartes 96 lápices entre 16 niños de modo que a cada uno le toque la misma cantidad? le plantea un problema, mientras que a estudiantes de niveles más altos esta pregunta sólo sugiere un ejercicio rutinario: "dividir ". Hacer ejercicios es muy valioso en el aprendizaje de las matemáticas: ayuda al estudiante a aprender conceptos, propiedades y procedimientos -entre otras cosas-, los cuales podrá aplicar cuando enfrente la tarea de resolver problemas.

Como se apunta anteriormente, la más grande contribución de Polya en la enseñanza de las matemáticas es su Método de Cuatro Pasos para 
resolver problemas, tales pasos son: 1. Entender el problema, 2. Configurar un plan, 3. Ejecutar el plan y 4. Mirar hacia atrás.

\section{Resultados y discusión}

El modelo pedagógico en el PEI del colegio no está establecido como tal, pero el plan de área de Matemáticas presenta una propuesta el cual se fundamenta en la teoría de Ausubel y su aprendizaje significativo, la teoría de Piaget (asimilación, adaptación desequilibrio, acomodación, equilibrio) y la teoría de Vygotsky sobre la zona de desarrollo próximo que es la diferencia entre lo que el niño o joven puede hacer solo y con ayuda de otra persona. Enmarcado en la construcción del conocimiento, donde compromete al educador a una búsqueda constante y actualización del conocimiento matemático para analizarlo y adaptarlo a las exigencias del avance de la ciencia y la tecnología.

Aportes de gran importancia para proponer estrategias que ayuden a mediar estas dificultades, enseñando al estudiante a resolver problemas matemáticos y de su entorno de manera creativa e interesante.

Los lineamientos curriculares de matemáticas contemplan la resolución de problemas como uno de los cinco procesos generales que están a lo largo de todas las actividades, más aún es el centro que abarca el contexto inmediato del estudiante a través de las situaciones problema en su diario vivir.

Este proceso permite el desarrollo de la actividad mental mediante la planeación e implementación de estrategias teniendo en cuenta el resolver, el encontrar resultados, el verificar e interpretar lo razonable, el modificar condiciones y el originar otros problemas, no solo desde el contexto matemático sino desde el contexto cotidiano del estudiante, buscando mejorar los resultados en las pruebas institucionales y las pruebas SABER.

La planeación de estas estrategias permiten al estudiante desde un ámbito agradable, cooperativo y eficaz resolver problemas matemáticos de la cotidianidad y sobre todo logrando ser competentes mediante "ambientes de aprendizaje enriquecidos por situaciones problema significativas $\mathrm{y}$ comprensivas, que posibiliten avanzar a niveles de competencia más y más complejos" (MEN, ESTANDARES BASICOS DE COMPETENCIAS EN MATEMATICAS) pero para esto es necesario que el docente reflexione, explore sobre su práctica en el aula y tenga una adecuada apropiación epistemológica del campo matemático.

El pensamiento numérico y sistemas numéricos el cual abarca lo operacional, las habilidades y destrezas numéricas, las estimaciones, los órdenes de magnitudes, etc... los estándares curriculares de matemáticas pretende con este pensamiento es que los estudiantes adquiera y comprendan el concepto de número a través de su utilidad en diferentes contextos significativos, en otras palabras cada punto de referencia que tiene el concepto de número se adquieren en el contexto y se evoluciona en los ambientes escolares y extraescolares en donde el estudiante se desenvuelve.

Según Polya, el docente debe guiar a sus estudiantes con una serie de preguntas a la solución de problemas matemáticos. En la etapa de la comprensión el docente debe proponer el problema con un nivel de dificultad de modo que sea interesante para el estudiante. En la etapa concebir un plan el docente guía al estudiante hacia una estrategia de solución basada en experiencias anteriores y presaberes. En la etapa de ejecución del plan el estudiante es quien examina los detalles y analiza los pasos realizados sean concretos. Finalmente, el cuarto paso tiene como objeto verificar el resultado permitiendo al estudiante afianzar sus conocimientos y desarrollar aptitudes para resolver otros problemas. Y sin olvidar que para lograr un aprendizaje significativo es de suma importancia ciertos factores para generar interés como: la creatividad, la motivación y la cooperación en la planeación de las estrategias.

Por ciertas razones, se busca que en los estudiantes haya una motivación por aprender partiendo desde 
la planeación y ejecución de clases estimulantes, agradables y sobre todo interesados en la participación activa y constante en su cotidianidad; por lo tanto este estudio pretende despertar el interés de los estudiantes de la Institución Educativa y generar un aprendizaje significativo desde nuevas estrategias que sirvan de apoyo en el desarrollo de competencias matemáticas, buscando mejorar los resultados en la pruebas institucionales y las pruebas SABER, es necesario insistir en mejorar el aprendizaje y en fortalecer la competencia de resolución de problemas a partir del aprendizaje de las operaciones básicas entre números naturales.

Así como la parte legal impulsa a obrar correctamente, se cuenta con un modelo esencial que hace parte importante de las obras educativas, que permite tener claridad del obrar como comunidad educativa. Por tanto, de acuerdo con el Proyecto Educativo Institucional el área de Matemáticas presenta la siguiente propuesta pedagógica.

Está enmarcado en la construcción del conocimiento, donde compromete al educador a una continua búsqueda y actualización del conocimiento matemático para analizarlo y adaptarlo a las exigencias del avance de la ciencia y la tecnología.

La enseñanza de las matemáticas se enfoca en el modelo constructivista basados en la teoría de Piaget (asimilación, adaptación desequilibrio, acomodación, equilibrio) y la teoría de Vygotsky sobre la zona de desarrollo próximo que es la diferencia entre lo que el niño o joven puede hacer solo y con ayuda de otra persona.

El docente con la renovación de sus conocimientos emplea estrategias para que el estudiante pueda también construir su propio conocimiento matemático desarrollando cada vez más sus procesos mentales (conceptualización, comprensión, análisis, síntesis y generalización), ya que sólo se alcanzan con una aplicación cada vez más amplia, más profunda y más rigurosa de la metodología científica.
El modelo constructivista percibe a los estudiantes como seres pensantes capaces de construir conceptos matemáticos mediante el razonamiento para resolver problemas a través de: Conjeturas. Razonamiento intuitivo o de inducción, por medio de la búsqueda de patrones. La deducción que se utiliza para comprobar las conjeturas junto a sus compañeros y el profesor, que actúa como mediador.

El constructivismo matemático se interesa por las condiciones en las cuales la mente realiza la construcción de los conceptos matemáticos, por la forma como los organiza en estructuras y por la aplicación que se les da, todo ello tiene consecuencias inmediatas en el papel que juega el estudiante en la generación y desarrollo de sus conocimientos. No basta con que el maestro haya hecho las construcciones mentales, cada estudiante necesita a su vez realizarlo, en eso nada ni nadie lo puede reemplazar.

La presente propuesta educativa se fundamenta en la Teoría de Ausubel (1973) y su aprendizaje significativo, la teoría de Piaget (1950) y Vygotsky (1979), además del Método Polya (1965) como se sustenta a continuación.

\section{La Teoría de Ausubel y su Aprendizaje Significativo}

Para este autor el aprender tiene una estrecha relación con la visión del aprendizaje, la que tiene relación con los procesos internos y cognitivos que pueda poseer una persona, teniendo éstas no solo una relación con sus respuestas a nivel externo.

Es con relación a esto, que se espera que los educadores tengan la capacidad de generar momentos en que los alumnos/as, sean capaces de asimilar los conocimientos que ya poseen, con nuevos conocimientos, de una manera accesible y clara, lo que facilita una mayor comprensión de estos, a través de la enseñanza receptivo significativa. Para esta teoría, el pilar fundamental es que los alumnos/as deben ser capaces de construir significados, los que serán elementos 
fundamentales para su enseñanza- aprendizaje. Para esto, se debe considerar que existen parámetros para que se genere un aprendizaje significativo.

En primera instancia, se debe procurar que exista una significatividad lógica en el contenido, es decir que los contenidos y estructuras del contenido que se espera que los niños y niñas adquieran tengan una concordancia e importancia real en su desarrollo. Además, el contenido debe tener una significatividad psicológica, refiriéndose a que se generen relaciones entre los aprendizajes previos que posee el niño o la niña, con relación al contenido que se encuentra adquiriendo. Y finalmente, el tercer parámetro fundamental es la motivación que debe existir, considerando que tanto el niño o la niña deben tener una disposición al nuevo aprendizaje.

Por lo tanto, se propone esta teoría a través de la resolución de problemas, ampliamente considerada conveniente y eje de la enseñanza de la matemática, teniendo en cuenta que, para este tipo de aprendizaje, Ausubel menciona que debe existir lo que denomina "actitud para el aprendizaje significativo", que se trata de una disposición por parte del estudiante para relacionar una tarea de aprendizaje sustancial y no arbitraria, con los aspectos relevantes de su propia estructura cognitiva. Este concepto que puede unirse al de motivación del aprendizaje, ligada durante el proceso de aprendizaje a "la comprensión posible por parte del alumno de la "significatividad" de lo que se aprende.

Para Ausubel la resolución de problemas es la forma de actividad o pensamiento dirigido en los que, tanto la representación cognoscitiva de la experiencia previa como los componentes de una situación problemática actual, son reorganizados, transformados o recombinados para lograr un objetivo diseñado; involucra la generación de estrategias que trasciende la mera aplicación de principios. Los problemas matemáticos entrañan un no saber, o bien una incompatibilidad entre dos ideas que se transforma en un obstáculo que se necesita atravesar. Esta solución se logrará utilizando básicamente un tipo de inteligencia: la lógico - matemática. La solución de problemas tiene valor porque cultiva procedimientos, métodos y heurísticas que son valiosos para la escuela y la vida.

\section{La Teoría de Piaget}

Piaget resalta la universalidad de la cognición y considera al contexto relativamente poco importante y escasamente influyente en los cambios cualitativos de la cognición. El niño es visto como constructor activo de su conocimiento $\mathrm{y}$, por lo tanto, del lenguaje.

Piaget presentó una teoría integrada del desarrollo cognitivo, que era universal en su aplicabilidad y fue caracterizada la estructura subyacente del pensamiento. Su aproximación es constructivista e interaccionista a la vez. Se proponen 2 mecanismos constructores de las estructuras cognitivas para tratar con entornos cada vez más complejos: la organización y la acomodación. Estos principios promueven además el estudio del desarrollo del lenguaje; teniendo claridad que éste se centraría en una expresión cada vez más clara y lógica del pensamiento y en una progresiva socialización, basada en la capacidad progresiva del niño para comprender puntos de vista ajenos (de lenguaje egocéntrico a social).

\section{Teoría de Vygotsky o de las Influencias Socioculturales}

Todo el proceso de la actividad de un sujeto está regulado socialmente. Mediación: "Toda actividad generadora de procesos mentales superiores" cuya fuente es tanto la herramienta material, como el sistema de símbolos, o el comportamiento de otro ser humano que interviene como mediador. Lo que Vygotsky explica es el desarrollo del niño y, para él, ese proceso está relacionado con la instrucción. Las aportaciones de Vygotsky pueden sintetizarse en los siguientes términos:

El desarrollo del individuo es resultado del proceso histórico y social donde el lenguaje 
resulta relevante; El conocimiento es resultado de la interacción social y en esa interacción con otros sujetos se constituye la conciencia. En este sentido, según Vygotsky el uso de símbolos es fundamental en el proceso de pensar de manera compleja. Uno de los símbolos más relevantes es el lenguaje escrito cuya complejidad es mayor, dice, que la del lenguaje hablado; La instrucción debe preceder al desarrollo y potenciarlo. Esa afirmación es contraria a la que sostenía Piaget para quien en cada estadio de desarrollo sólo pueden adquirirse determinados conocimientos. En ese sentido, Vygotsky planteó la categoría de zona de desarrollo próximo para resaltar la importancia del aprendizaje por la mediación por otros y el uso de herramientas, principalmente el lenguaje.

El uso de la crítica como parte del sistema de investigación. Vygotsky ha sido considerado constructivista porque concibió a los seres humanos "...como constructores permanentes en su entorno y de las representaciones de éste a través de su implicación en formas diferentes de actividad." (Wertsch, 1988, p. 196. Vid. García, 2000, p. 18).

\section{Conclusiones}

Para concluir se puede demostrar durante el desarrollo de la presente investigación, que las diversas dificultades que tienen los estudiantes del grado sexto para la resolución de problemas: al leer, analizar y solucionar situaciones problemáticas en los distintos conjuntos de numeración, se deben en cierto modo a la forma como se desarrollan estratégicamente los contenidos en el aula, por lo tanto los aportes de la autora demostraron una mejoría en la enseñanza y el aprendizaje de las matemáticas lo que permitirá a futuro mejores resultados en las pruebas institucionales y en las llamadas "PRUEBAS SABER" que se realizan anualmente en Colombia.

Asimismo, el presente estudio, deja a la disposición aportes científicos que permitan fortalecer las competencias matemáticas en los estudiantes del nivel de educación primaria, que a su vez se proyecten a otros grupos del mismo año y secciones afines.

\section{Referencias}

Ausubel, D. P. (1973). "Algunos aspectos psicológicos de la estructura del conocimiento". En Elam, S. (Comp.) La educación y la estructura del conocimiento. Investigaciones sobre el proceso de aprendizaje y la naturaleza de las disciplinas que integran el currículum. Ed. El Ateneo. Buenos Aires. Págs. 211-239.

Elliott, J. (1993). El cambio educativo desde la investigación-acción. Madrid: Ediciones Morata.

Kemmis, S. (1984). El curriculum: Más allá de una teoría de la reproducción. (2a. ed.) Madrid: Ediciones MORATA.

MEN, C. (2017). Índice Sintético de Calidad Educativa ISCE. Bogotá.

Polya, G. (1965). Cómo plantear y resolver problemas. Trillas.

Tamayo, M. (1994). Serie Aprender a Investigar: Módulo 2 - La investigación (3a ed.). Santa Fe de Bogotá: ICFES.

Tylor y Bogdan (1986) Introducción a los Métodos Cualitativos. España: Gedisa

Piaget, J. (1950). Introducción a la Epistemología Genética. T1: El pensamiento matemático. T2: El pensamiento físico. T3: El pensamiento biológico, el pensamiento psicológico y el pensamiento sociológico. Buenos Aires: Paidós, Reeditado en 1975.

Vigotsky, L., S. (1979). El desarrollo de los procesos psíquicos superiores. Barcelona: Edit. Crítica. 